\title{
Effects of dietary protein content and 2-hydroxy-4-methylthiobutanoic acid or DL-methionine supplementation on performance and oxidative status of broiler chickens
}

\author{
Quirine Swennen ${ }^{1} \dagger$, Pierre-André Geraert ${ }^{2}$, Yves Mercier $^{2}$, Nadia Everaert $^{1}$, Anneleen Stinckens ${ }^{1}$, \\ Hilke Willemsen ${ }^{1}$, Yue Li $^{1}$, Eddy Decuypere ${ }^{1}$ and Johan Buyse ${ }^{1_{*}}$ \\ ${ }^{1}$ Laboratory of Livestock Physiology, Immunology and Genetics, Department of Biosystems, K.U. Leuven, Kasteelpark \\ Arenberg 30, 3001, Leuven, Belgium \\ ${ }^{2}$ Adisseo France SAS, F-92160 Antony, France
}

(Received 23 July 2010 - Revised 4 April 2011 - Accepted 7 April 2011 - First published online 21 June 2011)

\section{Abstract}

Besides its typical role as an amino acid in protein synthesis, methionine is an important intermediate in methylation reactions. In addition, it can also be converted to cysteine and hence plays a role in the defence against oxidative stress. The present study was conducted to investigate further the role of DL-methionine (DLM) and its hydroxy analogue, DL-2-hydroxy-4-methylthiobutanoic acid (DL-HMTBA), on zootechnical performance and oxidative status of broiler chickens. Male broiler chickens were reared on two diets differing in crude protein (CP) content (low-protein, $18.3 \% v$. high-protein, $23 \cdot 2 \% \mathrm{CP}$ ) and were supplemented either with $0 \cdot 25 \%$ DLM or $0 \cdot 25 \%$ DL-HMTBA. Reducing the dietary protein content resulted in an impaired body weight gain $(P<0 \cdot 0001)$. However, supplementation of DL-HMTBA to the low-protein diet partially alleviated these negative effects $(P=0 \cdot 0003)$. This latter phenomenon could be explained by the fact that chickens fed DL-HMTBA-supplemented diets displayed a better antioxidant status as reflected in lower lipid peroxidation probably as a consequence of their higher hepatic concentrations of total and reduced glutathione compared with their DLM counterparts. On the other hand, within the high protein levels, uric acid might be an important antioxidant to explain the lower lipid peroxidation of high-protein DL-HMTBA-supplemented chickens. Hepatic methionine sulfoxide reductase-A gene expression was not significantly affected by the dietary treatments. In conclusion, the present study indicates that there are interactions between dietary protein content and supplementation of methionine analogues with respect to broiler performance and antioxidant status, also suggesting a causal link between these traits.

\section{Key words: DL-Methionine (analogue): Oxidative status: Broiler chickens}

Lysine and methionine are generally considered to be the first limiting amino acids in commercial wheat/maize-soyabeanbased broiler chicken diets. Methionine deficiency results in reduced protein accretion rate and feather growth as well as impaired immune competence ${ }^{(1,2)}$. The deficiency symptoms can be counteracted by adding chemically synthesised DL-methionine (DLM) or its hydroxy analogue, DL-2-hydroxy-4-methylthiobutanoic acid (DL-HMTBA), to the diet. In addition, the impact of DLM supplementation on animal performance interacts strongly with the crude protein (CP) content of the diet (e.g. Garcia Neto et $a l^{(3)}$ ). Supplementation of DLM to a $20 \%$ crude protein diet for broilers reduced their abdominal fat weight, whereas this was not the case when the diet contained $23 \% \mathrm{CP}^{(4)}$. Breast meat yield was increased and abdominal fat content was decreased with increasing DLM supplementation, and this response was more pronounced in chickens reared on a diet with a lower protein level $(20.5 \% \mathrm{CP})$ compared with a higher protein level $(26 \%)^{(5)}$. Fatufe \& Rodehutscord ${ }^{(6)}$ reported that the response to methionine in terms of feed intake, body weight gain and feed efficiency was more pronounced when this amino acid was added to a lowprotein (18.3\%) compared with an adequate-protein (22.9\%) ration in 8 - to 21 -d-old birds.

Besides the typical role of an amino acid as a building block in protein synthesis (anabolic function), methionine

\footnotetext{
Abbreviations: CP, crude protein; DL-HMTBA, DL-2-hydroxy-4-methylthiobutanoic acid; DLM, DL-methionine; FRAP, ferric reducing/antioxidant power; GSH, glutathione; GSSG, oxidised glutathione; Msr, methionine sulfoxide reductase; ROS, reactive oxygen species; $\mathrm{T}_{3}$, triiodothyronine; TBARS, thiobarbituric acid reacting substances.
}

*Corresponding author: Professor J. Buyse, fax +32 16 321994, email johan.buyse@biw.kuleuven.be

† Present address: Biomedical Research Institute, Hasselt University, Angoralaan Building C, 3590 Diepenbeek, Belgium. 
has several other prominent roles in metabolism. It is an important intermediate in methylation reactions and can also be converted to cysteine, which is required for the synthesis of glutathione (GSH) and taurine. It is well recognised that these sulphur compounds have a crucial role in the defence against oxidative stress (reviewed by Métayer et $a l .{ }^{(7)}$ ). Indeed, GSH and cysteine can act as direct scavengers of reactive oxygen species (ROS) and hence alleviate their deleterious effects on lipid, protein and DNA structures. Amino acid residues of proteins are very prone to oxidative damage, and this is particularly true for methionine residues. Fortunately, most cells are equipped with specific reductases: methionine sulfoxide reductase (Mrr)-A and -B. These enzymes catalyse the reduction of the two stereoisomeric oxidation products of methionine, namely methionine sulfoxide- $R$ and $-S$, back to methionine. In this way, the cyclic oxidation/reduction of methionine residues can even be considered as an important antioxidant defence system ${ }^{(8)}$. It is therefore hypothesised that extra methionine supplementation may be beneficial in alleviating oxidative stress by its ROS-scavenging activity.

Broiler chickens reared on diets with a low CP content are characterised by impaired body-weight gain, poor feed efficiency and augmented fat deposition ${ }^{(9)}$. In addition, intermediary metabolism and endocrine functioning are also disturbed (reviewed by Swennen et $a l .{ }^{(9)}$ ). As an example, plasma corticosterone levels of chickens reared on a lowprotein diet are chronically elevated ${ }^{(10)}$. This chronic exposure to high corticosterone concentrations enhances lipid peroxidation $^{(11,12)}$. Furthermore, chickens fed a diet with low protein content are also characterised by a significantly elevated metabolic rate, as is sometimes also reflected in higher plasma $3,3^{\prime}, 5$-triiodothyronine levels ${ }^{(9,13)}$. An increased metabolic rate is associated with higher oxygen consumption and hence an augmented ROS production can be inferred. Therefore, the aims of the present study were as follows:

(1) to elaborate further on the interaction between dietary methionine supplementation and the CP content of the basal diet on broiler chicken performance;

(2) to assess whether a low-protein diet (18.3 v. 23.2\% $\mathrm{CP})$ is unfavourable in terms of redox balance and to assess whether supplementation with methionine sources could alleviate the deleterious effects of a redox imbalance;

(3) to assess whether dietary supplementation with an equimolar amount of the DL-hydroxy analogue DL-HMTBA elicits the same effects as synthetic DLM on bird performance, intermediary metabolism, endocrine status and redox balance. Because DL-HMTBA has been shown to be more efficiently converted to cysteine and taurine than L-methionine ${ }^{(14)}$, this could lead to an ameliorated antioxidant action.

\section{Materials and methods}

The experimental protocol was approved by the Ethics Commission for Experimental Use of Animals of the K. U. Leuven.

\section{Experimental set-up}

Newly hatched male chicks ( $n$ 310; Ross 308) were obtained from a local hatchery (Belgabroed, Merksplas, Belgium). All chicks were reared in floor pens with wood shavings as litter. Temperature was set at $34^{\circ} \mathrm{C}$ for day-olds and gradually decreased to $20^{\circ} \mathrm{C}$ at 5 weeks of age. The lighting schedule provided $23 \mathrm{~h}$ of light per $\mathrm{d}$.

Until $14 \mathrm{~d}$ of age, all chicks received a commercial starter diet (22.3\% CP and $12.7 \mathrm{MJ}$ metabolisable energy $/ \mathrm{kg}$ ) ad libitum. At $14 \mathrm{~d}$ of age, the chickens were divided into four treatment groups of seventy-five chickens receiving one of the four experimental diets ad libitum (Table 1). The four treatment groups were divided randomly over the poultry house with three pens per treatment group and twenty-five broiler chickens per pen. The levels of soyabean, soyabean meal, soya oil and celite were manipulated to create changes in protein and fat content and to keep the metabolisable energy level similar for both diets $(12.5 \mathrm{MJ}$ metabolisable energy $/ \mathrm{kg})$. This resulted in two isoenergetic diets with a high (23.2\%) or a low (18.3\%) CP level. For both diets, the National Research Council recommendations (1994) for essential amino acids were met. The low- and high-CP diets were supplemented either with $0 \cdot 25 \%$ DLM or with $0 \cdot 25 \%$ DL-HMTBA (Adisseo SAS, Antony, France), resulting in a total of four diets.

\section{Measurements and sampling}

Individual body weights and feed intake per pen were recorded on a weekly basis. At 4 and 6 weeks of age, fifteen broiler chickens per treatment group (five chickens per pen) were euthanised, and the liver, right pectoralis major of the breast, abdominal fat pad and heart were excised and weighed. Samples of the liver were immediately snap frozen in liquid $\mathrm{N}_{2}$ and stored at $-80^{\circ} \mathrm{C}$. In addition, blood samples were collected from a wing vein using a heparinised syringe and kept on ice. After centrifugation, plasma was stored in a frozen condition $\left(-20^{\circ} \mathrm{C}\right)$ until the analysis for hormone and metabolite concentrations.

\section{Plasma analyses}

Plasma triiodothyronine $\left(\mathrm{T}_{3}\right)$ concentrations were measured by RIA as described by Darras et al. ${ }^{(15)}$. The antiserum for $\mathrm{T}_{3}$ was purchased from Byk-Belga (Diegem, Belgium). Plasma corticosterone levels were measured using a commercially available double antibody RIA kit (IDS Limited, Boldon, UK). The detection limit of the assay was $0.39 \mathrm{ng} / \mathrm{ml}$.

Plasma uric acid (VetTest 9820378) concentrations were determined using the VetTest 8008 analyser (Idexx Laboratories, Inc., Westbrook, ME, USA). The apparatus is based on dry chemical technology and colorimetric reaction. Sample analysis is carried out on selective testing discs (Idexx Laboratories, Inc.) by means of a laser reading the bar codes.

Plasma superoxide dismutase (SOD) activity was assessed using the SOD assay kit (Dojindo Molecular Technologies Inc., Rockville, MD, USA) according to the manufacturer's recommendations using a microplate reader $\left(\right.$ Victor $^{3} \mathrm{~V}^{\mathrm{TM}}$ 
Table 1. Diet components and calculated and analysed contents of the experimental diets

\begin{tabular}{|c|c|c|c|c|}
\hline & \multicolumn{2}{|c|}{ High-protein } & \multicolumn{2}{|c|}{ Low-protein } \\
\hline & DLM & DL-HMTBA & DLM & DL-HMTBA \\
\hline \multicolumn{5}{|l|}{ Ingredients (\%) } \\
\hline Soyabean meal & $41 \cdot 1$ & $41 \cdot 1$ & - & - \\
\hline Full-fat soyabeans & - & - & $37 \cdot 6$ & $37 \cdot 6$ \\
\hline Soya oil & 7 & 7 & 2.58 & 2.58 \\
\hline Maize & 51 & 51 & 51 & 51 \\
\hline Trace mineral-vitamin premix ${ }^{*}$ & 0.5 & 0.5 & 0.5 & 0.5 \\
\hline Phytase premix & 0.1 & 0.1 & 0.1 & $0 \cdot 1$ \\
\hline Celite† & - & - & 7.86 & 7.86 \\
\hline \multicolumn{5}{|c|}{ Calculated energy and nutrient content } \\
\hline Metabolisable energy (MJ/kg) & $12 \cdot 5$ & $12 \cdot 5$ & $12 \cdot 5$ & $12 \cdot 5$ \\
\hline $\mathrm{CP}(\%)$ & $23 \cdot 2$ & $23 \cdot 2$ & $18 \cdot 3$ & $18 \cdot 3$ \\
\hline DL-Met $(99 \%) \ddagger$ & 0.29 & 0.036 & 0.30 & 0.098 \\
\hline DL-HMTBA $(88 \%) \ddagger$ & - & 0.28 & - & 0.28 \\
\hline Met equivalence (\%) & 0.64 & 0.64 & 0.59 & 0.59 \\
\hline Met + Cys & 1.02 & 0.73 & 0.80 & 0.59 \\
\hline Met equivalence + Cys (\%) & 1.02 & 1.02 & 0.89 & 0.89 \\
\hline \multicolumn{5}{|c|}{ Analysed energy and nutrient content } \\
\hline Metabolisable energy $(\mathrm{MJ} / \mathrm{kg})$ & 12.46 & $12 \cdot 47$ & 12.45 & $12 \cdot 45$ \\
\hline CP (\%) & $22 \cdot 3$ & 21.3 & $18 \cdot 1$ & $18 \cdot 7$ \\
\hline DL-Met (\%) & 0.62 & 0.38 & 0.53 & 0.38 \\
\hline DL-HMTBA (\%) & - & 0.2 & - & 0.2 \\
\hline Lys (\%) & 1.23 & $1 \cdot 19$ & 0.95 & 1.01 \\
\hline $\operatorname{Arg}(\%)$ & 1.76 & 1.64 & $1 \cdot 3$ & 1.42 \\
\hline Thr (\%) & 0.88 & 0.73 & 0.85 & 0.75 \\
\hline Val (\%) & 1.08 & 1.01 & 0.79 & 0.88 \\
\hline $\mathrm{Ca}(\%)$ & 0.85 & 0.85 & 0.85 & 0.85 \\
\hline Non-phytate P (\%) & 0.44 & 0.44 & 0.44 & 0.44 \\
\hline $\mathrm{Na}(\%)$ & 0.16 & 0.16 & 0.16 & 0.16 \\
\hline
\end{tabular}

DLM, DL-methionine; DL-HMTBA, DL-2-hydroxy-4-methylthiobutanoic acid; CP, crude protein, NRC, National Research Council.

* Premix supplied the following amount of vitamins and minerals per kg of diet: vitamin A, $12000 \mathrm{IU}$; vitamin $\mathrm{D}_{3}, 3000 \mathrm{IU}$; vitamin $\mathrm{E}, 50 \mathrm{IU}$; vitamin $\mathrm{K}_{3}, 2.5 \mathrm{mg}$; vitamin $\mathrm{B}_{1}, 2.2 \mathrm{mg}$; vitamin $\mathrm{B}_{2}, 0.035 \mathrm{mg}$; vitamin niacin, $38 \mathrm{mg}$; folic acid, $1 \mathrm{mg}$; biotin, $0.2 \mathrm{mg}$; choline-Cl, $650 \mathrm{mg}$; $\mathrm{Fe}, 45 \mathrm{mg}$; Cu, $25 \mathrm{mg}$; Mn, $60 \mathrm{mg}$; Co, $1 \mathrm{mg} ; \mathrm{Zn}, 70 \mathrm{mg}$; I, $2 \mathrm{mg}$; Se, $0.4 \mathrm{mg}$; ethoxyquin, $35 \mathrm{mg}$; butylated hydroxytoluene, $25 \mathrm{mg}$.

$\dagger$ Celite is an energy-inert material used to obtain isoenergetic diets.

‡ For all diets, the NRC recommendations (1994) for essential amino acid were met. Specifically for methionine, DLM (Adisseo France SAS) was added to the diets irrespective of protein level to achieve the NRC recommendations of $4 \mathrm{~g} \mathrm{Met} / \mathrm{kg}$. Finally, the low- and high-CP diets were supplemented either with DLM $(0.25 \%)$ or with DL-HMTBA $(0.25 \%)$, resulting in a total of four diets.

Multilabel Counter 1420, PerkinElmer, Waltham, MA, USA). Plasma lipid peroxidation was estimated by spectrophotometric determination of thiobarbituric acid reacting substances (TBARS) with the modified method of Lin et al. ${ }^{(11,12)}$. TBARS were expressed as nmol malondialdehyde (MDA) per ml plasma. Plasma ferric reducing/antioxidant power (FRAP) was determined using a room temperature assay with a 5 min time window as described by Lin et $a l^{(11,12)}$. Ceruloplasmin levels in the plasma were measured with the $p$-phenylenediamine endpoint $\operatorname{method}^{(16)}$.

\section{Hepatic glutathione analysis}

For the quantification of total GSH and oxidised GSH or GSH disulfide (GSSG) concentrations in the liver samples, an enzymatic recycling method using GSH reductase was used. The preparation of the samples and the colorimetric endpoint measuring method was carried out according to the manufacturer's recommendation (Glutathione assay kit, no. 703002; Cayman Chemical Company, Ann Arbor, MI, USA). Reduced GSH levels could then be calculated by subtracting twice the GSSG concentration from the total GSH concentration. Total GSH, GSSG and reduced GSH were expressed relative to the protein concentration of the liver samples used.
The total protein concentration of the samples was determined by using the Pierce BCA Protein Assay (Thermoscientific, Rockford, IL, USA).

\section{Reverse transcription and RT-PCR}

The liver tissue was homogenised with a homogeniser (Ultra-turrax, Janke \& Kunkel, Staufen, Germany), and RNA was extracted using TRIzol reagent (Invitrogen, Life Technologies, Paisley, UK), according to the manufacturer's recommendations. Total RNA concentration was quantified and its integrity was verified by means of an UV spectrophotometer.

Total RNA $(2 \mu \mathrm{g})$ was reverse transcribed using Avian Myeloblastosis Virus-RT (Promega, Catalogue no. M5108, Madison, WI, USA).

The primers for Msr-A, GSH synthetase and GSH reductase were designed using Primer premier 5.0 using the GenBank sequences for Msr-A (accession no. XM_420035.2), the GenBank sequences for GSH synthetase (accession no. XM_425692.2) and the Ensembl sequences for GSH reductase (accession no. ENSGALT00000016706), respectively. The primer that was used for the reference gene glucose-6phosphate dehydrogenase (accession no. AI981686) was 
from De Boever et al. ${ }^{(17)}$. Desalted primers for the target gene Msr-A (forward primer, 5'-TGA CTT TGG CAC TCA GTA TCG C-3'; reverse primer, $5^{\prime}$-GGC ACC AAA ACC GCT TTC T-3'), GSH synthetase (forward primer, 5'-AGA AGG AGG AGG GAA CAA CC- $3^{\prime}$; reverse primer, 5'-GCC TGA CAT AGA CAC CGA AA-3'), GSH reductase (forward primer, 5'-CCA TGT GGT GGT GGA CGA GT-3'; reverse primer, 5'-GCA GAG GTA GGG TGG ATA GCA-3') and glucose-6-phosphate dehydrogenase (forward primer, 5'-CGG GAA CCA AAT GCA CTT CGT-3'; reverse primer, 5'-CGC TGC CGT AGA GGT ATG GGA-3') were purchased commercially (Invitrogen, Zwolle, The Netherlands).

Real-time PCR to quantify Msr-A, GSH synthetase and GSH reductase mRNA was performed using the ABI Prism 7700 Sequence Detector (ABI PRISM ${ }^{\mathrm{TM}}$; Life Technologies, Carlsbad, CA, USA.

The sequence detection software supplied with the 7700 Sequence Detector was used to analyse the raw data from the real-time reading of the fluorescence and to calculate the cycle threshold value for each reaction. The cycle threshold values were converted into a standard curve, to which each sample (average of three cycle threshold values) was compared to calculate the starting template concentrations. Expression levels of Msr-A, GSH synthetase and GSH reductase in each sample were normalised to the expression levels of the reference gene glucose-6-phosphate dehydrogenase. The average value of the high $\mathrm{CP}-\mathrm{DL}-\mathrm{HMTBA}$ group was set at 1 and the average value of the other three groups was expressed relative to the high $\mathrm{CP}-\mathrm{DL}-\mathrm{HMTBA}$ group.

\section{Statistical analysis}

All data were analysed by two-factor ANOVA, with dietary protein level $(23.2$ or $18.3 \% \mathrm{CP}$ ) and supplement source (DLM or DL-HMTBA) as classification variables (SAS version 9.1; SAS Institute, Inc., Cary, NC, USA). If a significant
$(P<0.05)$ overall effect of the model was discerned, treatment means were separated by Tukey's test. Statistical significance is accepted when $P<0 \cdot 05$.

\section{Results}

Body weight, feed intake and proportional organ weights

Data on body weight and proportional breast muscle, abdominal fat pad, heart and liver weights collected at 4 weeks of age are summarised in Table 2. It was clear that the chickens reared on the low-protein diets attained a significantly lower body weight (protein effect, $P<0.0001$ ) at 4 weeks of age, irrespective of the supplement source (DLM or DL-HMTBA). These reduced body weights of the low CP-fed broilers were also associated with significantly (protein effect, $P<0 \cdot 0001$ ) lower proportional (right) breast muscle weights. As expected, proportional abdominal fat pad weights of chickens fed the low-protein diets were significantly higher than those of chickens reared on a high-protein diet (protein effect, $P<0.0001$ ). The supplement source had no differential effect on body weight or on proportional breast and abdominal fat pad weight. Chickens fed a low-protein diet had significantly higher proportional heart weights (protein effect, $P=0 \cdot 041$ ), but this effect of dietary protein on the percentage of heart weight was also dependent on the supplement source (protein $\times$ supplement source effect, $P=0 \cdot 02$ ). Indeed, chickens reared on a low-protein diet and receiving DLM as a supplement were characterised by a significantly higher proportional heart weight compared with that of their highprotein DLM-fed counterparts. DL-HMTBA-supplemented chickens had an intermediate proportional heart weight. The (proportional) liver weights were not different between the treatment groups.

The retardation in body-weight gain due to low dietary $\mathrm{CP}$ content (protein effect, $P<0 \cdot 0001$ ) persisted at 6 weeks of age

Table 2. Body weights and proportional organ and tissue weights of 4-week-old broiler chickens raised on diets with low $(18.3 \%)$ or high $(23.2 \%)$ crude protein (CP) content and supplemented with $0.25 \%$ DL-methionine (DLM) or $0.25 \%$ DL-2hydroxy-4-methylthiobutanoic acid (DL-HMTBA)

(Mean values with their pooled standard errors, $n$ 15/dietary treatment)

\begin{tabular}{|c|c|c|c|c|c|c|}
\hline & \multirow[b]{2}{*}{ Met source } & \multirow[b]{2}{*}{ BW (g) } & \multicolumn{4}{|c|}{ Proportional weight } \\
\hline & & & Liver (\%) & Heart (\%) & Abdominal fat (\%) & Breast muscle (\%) \\
\hline \multicolumn{7}{|l|}{ Protein level (\%) } \\
\hline \multirow[t]{2}{*}{$18 \cdot 3$} & DLM & 1156 & 2.48 & $0.37^{\mathrm{a}}$ & 1.47 & 7.44 \\
\hline & DL-HMTBA & 1145 & $2 \cdot 41$ & $0.34^{a, b}$ & 1.41 & $7 \cdot 31$ \\
\hline \multirow[t]{2}{*}{$23 \cdot 2$} & DLM & 1428 & $2 \cdot 69$ & $0.32^{\mathrm{b}}$ & $1 \cdot 11$ & $8 \cdot 17$ \\
\hline & DL-HMTBA & 1424 & 2.59 & $0.34^{a, b}$ & 1.09 & 8.57 \\
\hline Pooled SEM & & 25 & 0.05 & 0.006 & 0.04 & 0.11 \\
\hline \multicolumn{7}{|l|}{ Main effects } \\
\hline \multirow[t]{2}{*}{ Protein level } & $18 \cdot 3$ & $1150^{\mathrm{b}}$ & $2 \cdot 41$ & 0.35 & $1.43^{\mathrm{a}}$ & $7 \cdot 38^{b}$ \\
\hline & $23 \cdot 2$ & $1426^{\mathrm{a}}$ & $2 \cdot 64$ & 0.33 & $1 \cdot 10^{\mathrm{b}}$ & $8.37^{\mathrm{a}}$ \\
\hline \multirow[t]{2}{*}{ Met source } & DLM & 1292 & 2.58 & 0.35 & 1.29 & $7 \cdot 81$ \\
\hline & DL-HMTBA & 1285 & $2 \cdot 47$ & 0.34 & $1 \cdot 24$ & 7.95 \\
\hline \multicolumn{7}{|l|}{ Probabilities } \\
\hline & Protein level & $<0.0001$ & NS & 0.041 & $<0.0001$ & $<0.0001$ \\
\hline & Met source & NS & NS & NS & NS & NS \\
\hline & Interaction & NS & NS & 0.02 & NS & NS \\
\hline
\end{tabular}

${ }^{a, b}$ Mean values with unlike superscript letters within a column were significantly different according to corresponding probabilities. 
Table 3. Body weights and proportional organ and tissue weights of 6-week-old broiler chickens raised on diets with low $(18.3 \%)$ or high $(23.2 \%)$ crude protein (CP) content and supplemented with $0.25 \%$ DL-methionine (DLM) or $0.25 \%$ DL-2-hydroxy-4-methylthiobutanoic acid (DL-HMTBA)

(Mean values with pooled standard errors, $n$ 15/dietary treatment)

\begin{tabular}{|c|c|c|c|c|c|c|}
\hline & \multirow[b]{2}{*}{ Met source } & \multirow[b]{2}{*}{$\mathrm{BW}(\mathrm{g})$} & \multicolumn{4}{|c|}{ Proportional weight } \\
\hline & & & Liver (\%) & Heart (\%) & Abdominal fat (\%) & Breast muscle (\%) \\
\hline \multicolumn{7}{|l|}{ Protein level (\%) } \\
\hline \multirow[t]{2}{*}{$18 \cdot 3$} & DLM & 2024 & 1.76 & $0.32^{a}$ & 1.64 & $8 \cdot 19$ \\
\hline & DL-HMTBA & 2301 & 1.92 & $0 \cdot 28^{b}$ & 1.68 & $8 \cdot 28$ \\
\hline \multirow[t]{2}{*}{$23 \cdot 2$} & DLM & 2492 & 1.89 & $0 \cdot 28^{b}$ & $1 \cdot 12$ & $9 \cdot 31$ \\
\hline & DL-HMTBA & 2707 & $2 \cdot 02$ & $0 \cdot 30^{a, b}$ & 1.03 & 8.99 \\
\hline Pooled SEM & & 46 & 0.03 & 0.004 & 0.06 & 0.12 \\
\hline \multicolumn{7}{|l|}{ Main Effects } \\
\hline \multirow[t]{2}{*}{ Protein level } & $18 \cdot 3$ & $2153^{b}$ & $1.84^{b}$ & 0.30 & $1.66^{a}$ & $8 \cdot 23^{b}$ \\
\hline & $23 \cdot 2$ & $2599^{a}$ & $1.96^{\mathrm{a}}$ & 0.29 & $1.07^{b}$ & $9 \cdot 16^{\mathrm{a}}$ \\
\hline \multirow[t]{2}{*}{ Met source } & DLM & $2258^{b}$ & $1.83^{b}$ & 0.30 & $1 \cdot 38$ & $8 \cdot 75$ \\
\hline & DL-HMTBA & $2518^{a}$ & $1.98^{a}$ & 0.29 & $1 \cdot 33$ & 8.63 \\
\hline \multicolumn{7}{|l|}{ Probabilities } \\
\hline & Protein level & $<0.0001$ & 0.042 & NS & $<0.0001$ & $<0.0001$ \\
\hline & Met source & 0.0030 & 0.011 & NS & NS & NS \\
\hline & Interaction & NS & NS & 0.0012 & NS & NS \\
\hline
\end{tabular}

${ }^{a, b}$ Mean values with unlike superscript letters within a column were significantly different according to corresponding probabilities.

(Table 3). However, supplementation of the low-protein diet with DL-HMTBA alleviated this growth-depressing effect of low dietary protein to some extent, as these chickens were able to attain a significantly higher average slaughter weight compared with that of their low-protein DLM-supplemented counterparts (supplement source effect, $P=0 \cdot 003$ ). Chickens reared on the low-protein diets also had the lowest proportional breast muscle weights (protein effect, $P<0.0001$ ) and lowest proportional liver weights (protein effect, $P=0 \cdot 042$ ). However, the proportional liver weights of the DL-HMTBAsupplemented chickens were, on average, higher than those of the DLM-supplemented chickens (supplement source effect, $P=0 \cdot 011$ ). The stimulatory effect of low dietary protein content (protein effect, $P<0 \cdot 0001$ ) on proportional abdominal fat pad deposition was even more pronounced at slaughter age, irrespective of DLM or DL-HMTBA supplementation.
In agreement with the observations at 4 weeks of age, the low-protein DLM-fed chickens were characterised by the highest proportional heart weights compared with the other treatment groups (protein $\times$ supplement source interaction, $P=0 \cdot 0012)$.

Chickens reared on the low-protein diets consumed, on average, less feed than their counterparts fed the high-protein diets, irrespective of the supplement source (data not shown). The reducing effect of dietary protein content on feed intake was significant at weeks $3(P=0 \cdot 0068)$ and $6(P=0 \cdot 0053)$.

\section{Redox balance}

At 4 weeks of age, lipid peroxidation - as reflected in plasma TBARS levels - was highest (supplement source effect, $P=0 \cdot 011$ ) for the DLM-supplemented chickens (Table 4).

Table 4. Plasma thiobarbituric acid reacting substances (TBARS), ferric reducing/antioxidant power (FRAP), ceruloplasmin and uric acid levels of 4-week-old broiler chickens raised on diets with low (18.3\%) or high $(23.2 \%)$ crude protein (CP) content and supplemented with $0.25 \%$ DL-methionine (DLM) or $0.25 \%$ DL-2-hydroxy-4-methylthiobutanoic acid (DL-HMTBA)

(Mean values with their pooled standard errors, $n$ 15/dietary treatment)

\begin{tabular}{|c|c|c|c|c|c|}
\hline & Met source & TBARS (nmol/l) & $\operatorname{FRAP}\left(\mu \mathrm{mol} \mathrm{Fe}{ }^{2+} / \mathrm{l}\right)$ & Ceruloplasmin (U/I) & Uric acid (mg/l) \\
\hline \multicolumn{6}{|l|}{ Protein level (\%) } \\
\hline \multirow[t]{2}{*}{$18 \cdot 3$} & DLM & 3.89 & $1981^{a}$ & 74.9 & $57 \cdot 3$ \\
\hline & DL-HMTBA & 2.75 & $1506^{c}$ & 55.4 & $57 \cdot 0$ \\
\hline \multirow[t]{2}{*}{$23 \cdot 2$} & DLM & 4.09 & $1976^{a}$ & 114 & $72 \cdot 4$ \\
\hline & DL-HMTBA & 1.83 & $1750^{\mathrm{b}}$ & $64 \cdot 8$ & $76 \cdot 7$ \\
\hline Pooled SEM & & 0.33 & 36 & 4.92 & $2 \cdot 2$ \\
\hline \multicolumn{6}{|l|}{ Main effects } \\
\hline \multirow{2}{*}{ Protein level } & $18 \cdot 3$ & 3.32 & 1744 & $65 \cdot 1^{\mathrm{b}}$ & $57 \cdot 3^{\mathrm{b}}$ \\
\hline & $23 \cdot 2$ & 2.96 & 1863 & $89 \cdot 7^{\mathrm{a}}$ & $74.5^{\mathrm{a}}$ \\
\hline \multirow[t]{2}{*}{ Met source } & DLM & $3.99^{\mathrm{a}}$ & 1979 & $94.7^{\mathrm{a}}$ & 64.9 \\
\hline & DL-HMTBA & $2 \cdot 29^{b}$ & 1628 & $60 \cdot 1^{\mathrm{b}}$ & $66 \cdot 8$ \\
\hline \multicolumn{6}{|l|}{ Probabilities } \\
\hline & Protein level & NS & NS & 0.0037 & $<0.0001$ \\
\hline & Met source & 0.011 & $<0.0001$ & $<0.0001$ & NS \\
\hline & Interaction & NS & 0.0021 & NS & NS \\
\hline
\end{tabular}

${ }^{a, b, c}$ Mean values with unlike superscript letters within a column were significantly different according to corresponding probabilities. 
Table 5. Plasma thiobarbituric acid reacting substances (TBARS), ferric reducing/antioxidant power (FRAP), ceruloplasmin and uric acid levels of 6-week-old broiler chickens raised on diets with low (18.3\%) or high (23.2\%) crude protein (CP) content and supplemented with $0.25 \%$ DL-methionine (DLM) or $0.25 \%$ DL-2-hydroxy4-methylthiobutanoic acid (DL-HMTBA)

(Mean values with their pooled standard errors, $n$ 15/dietary treatment)

\begin{tabular}{|c|c|c|c|c|c|}
\hline & Met source & TBARS (nmol/l) & $\operatorname{FRAP}\left(\mu \mathrm{mol} \mathrm{Fe} e^{2+} / \mathrm{l}\right)$ & Ceruloplasmin (U/l) & Uric acid (mg/l) \\
\hline \multicolumn{6}{|l|}{ Protein level (\%) } \\
\hline \multirow[t]{2}{*}{$18 \cdot 3$} & DLM & $6 \cdot 98^{a, b}$ & $934^{a, b}$ & $40 \cdot 3$ & $38 \cdot 7^{b}$ \\
\hline & DL-HMTBA & $9 \cdot 15^{\mathrm{a}}$ & $793^{b}$ & $29 \cdot 2$ & $39.0^{\mathrm{b}}$ \\
\hline \multirow[t]{2}{*}{$23 \cdot 2$} & DLM & $8.72^{\mathrm{a}}$ & $806^{a, b}$ & 38.3 & $39.6^{\mathrm{b}}$ \\
\hline & DL-HMTBA & $4.43^{b}$ & $964^{\mathrm{a}}$ & $32 \cdot 0$ & $68.5^{\mathrm{a}}$ \\
\hline Pooled SEM & & 0.46 & 24 & 1.47 & $2 \cdot 7$ \\
\hline \multicolumn{6}{|l|}{ Main effects } \\
\hline \multirow[t]{2}{*}{ Protein level } & $18 \cdot 3$ & 7.99 & 866 & $35 \cdot 1$ & $38.8^{\mathrm{b}}$ \\
\hline & 23.2 & 6.57 & 885 & $35 \cdot 2$ & $54 \cdot 1^{\mathrm{a}}$ \\
\hline \multirow{2}{*}{ Met source } & DLM & $7 \cdot 85$ & 868 & $39 \cdot 3^{\mathrm{a}}$ & $39.2^{b}$ \\
\hline & DL-HMTBA & 6.63 & 885 & $30 \cdot 7^{b}$ & $54 \cdot 8^{a}$ \\
\hline \multicolumn{6}{|l|}{ Probabilities } \\
\hline & Protein level & NS & NS & NS & 0.0007 \\
\hline & Met source & NS & NS & 0.0031 & 0.0012 \\
\hline & Interaction & 0.0002 & 0.0012 & NS & 0.0014 \\
\hline
\end{tabular}

${ }^{a, b}$ Mean values with unlike superscript letters within a column were significantly different according to corresponding probabilities.

DL-HMTBA-supplemented chickens displayed a lower plasma FRAP capacity compared with that of the DLM-supplemented chickens (supplement source effect, $P<0 \cdot 0001$ ). This supplement source effect on plasma FRAP capacity was even more pronounced with the low-protein diet (protein $X$ supplement source interaction, $P=0 \cdot 0021)$. DLM-supplemented chickens displayed overall higher plasma ceruloplasmin levels (supplement source effect, $P<0 \cdot 0001$ ) at 4 weeks of age compared with their DL-HMTBA counterparts. Chickens reared on a high-protein diet also had, on average, higher plasma ceruloplasmin levels (protein effect, $P=0 \cdot 0037$ ). Plasma uric acid concentrations were only affected by dietary protein content (protein effect, $P<0.0001$ ), as chickens reared on a low-protein diets were characterised by significantly lower plasma uric acid levels, irrespective of the supplement source.

At 6 weeks of age, a significant $(P=0.0002)$ protein $\times$ supplement source interaction was observed as significant differences in plasma TBARS levels were observed between the DL-HMTBA- and DLM-supplemented chickens but only when reared on a high-CP diet (Table 5). A significant protein $\times$ supplement source interaction $(P=0.0012)$ was also present for plasma FRAP capacity: DL-HMTBA-supplemented chickens were characterised by a higher plasma FRAP capacity but only when a high-CP diet was provided. Circulating ceruloplasmin levels were clearly affected by the supplement source $(P=0 \cdot 0031)$. Indeed, plasma obtained from DL-HMTBA-supplemented chickens contained significantly lower concentrations of ceruloplasmin compared with that from DLM-supplemented chickens, regardless of the dietary protein level. Higher dietary CP content was associated with significantly (protein effect, $P=0.0007$ ) higher plasma uric acid levels. However, this effect was only observed for DL-HMTBA-supplemented chickens (supplement source effect, $P=0.0012)$, resulting in a significant $(P=0.0014)$ protein $\times$ supplement source interaction.
Plasma SOD activity was not significantly influenced by either the dietary protein level or the supplement source at 4 weeks of age (data not shown). At 6 weeks of age, DL-HMTBA-supplemented chickens had, on average, higher plasma SOD activities compared with those of their DLMsupplemented counterparts, but this difference was not statistically discernable at the $5 \%$ level.

Statistical analysis revealed a tendency $(P=0.062)$ towards an interaction between protein level and supplement source for hepatic total GSH levels of 6-week-old chickens. Indeed, low-protein DL-HMTBA-supplemented chickens had somewhat higher amounts of total GSH compared with other treatment groups. Furthermore, there was also a tendency ( $P=0.095)$ towards a supplement source effect on hepatic reduced GSH amounts as DL-HMTBA-supplemented chickens had, on average, higher amounts of the reduced form of GSH. Statistical analysis did not reveal any significant effects on GSH synthetase, glutathione reductase or Msr-A gene expression in the livers from 6-week-old chickens (Table 6).

\section{Plasma hormones}

At 4 weeks of age, chickens reared on the low-protein diets had, on average, higher plasma $\mathrm{T}_{3}$ levels (protein effect, $P=0.0030$ ) than their counterparts fed the high-protein diets (Table 7). DL-HMTBA supplementation significantly (supplement source effect, $P=0.0006$ ) reduced circulating $\mathrm{T}_{3}$ levels of 4 -week-old chickens on the high-protein diets. However, at 6 weeks of age, higher $\mathrm{T}_{3}$ concentrations were measured in the plasma obtained from low-protein DLHMTBA-supplemented chickens compared with those from low-protein DLM-fed chickens (protein $\times$ supplement source interaction, $P=0 \cdot 012$ ).

In general, plasma corticosterone levels were significantly lower for the DL-HMBTA-supplemented chickens (at 4 weeks 
of age: supplement source effect, $P=0.025)$ compared with those of their DLM-supplemented counterparts. At 6 weeks of age, this observation was especially true for DL-HMTBAsupplemented chickens reared on a high-protein diet (protein $\times$ supplement source interaction, $P=0 \cdot 013$ ).

\section{Discussion}

\section{Zootechnical performance and plasma bioindicators}

Body-weight gain was significantly depressed by feeding a diet with a reduced $(18.3 v .23 \cdot 2 \%) \mathrm{CP}$ content. This observation is in agreement with many other studies (reviewed by Swennen et $\left.a l .{ }^{(9)}\right)$.

The higher proportional abdominal fat pad weights of the chickens reared on the low-protein diets are in agreement with previous studies and follow from the involuntary overconsumption of energy compared with protein (reviewed by Swennen $\left.e t a l .{ }^{(9)}\right)$. This excess energy is then partially deposited as fat and partially lost as heat ${ }^{(13)}$.

Another clear effect is the marked reduction in plasma uric acid concentrations in plasma obtained from chickens reared on a low-protein diet. This phenomenon has been observed several times before (reviewed by Swennen et al.$^{(9)}$ ) and illustrates the improved efficiency of nitrogen utilisation (better dietary protein retention and less protein degradation/amino acid oxidation) with lower protein intake.

However, DL-HMTBA supplementation to the low-protein diet partially alleviated the growth-depressing effect of the low-protein diet. This is in contrast with the statement of Sauer et $a l .{ }^{(1)}$ from their meta-analysis study that on an equimolar basis, the biological efficiency of methionine hydroxy analogue-free acid is $81 \%$ of the value for DLM for daily body-weight gain. On the other hand, using multiple regression models, Vasquez-Anon et al. ${ }^{(18)}$ did not observe a statistically significant difference in predicted responses for body-weight gain between DLM and DL-HMTBA, and these authors even mentioned a trend $(P \leq 0 \cdot 1)$ for a higher peak body-weight gain response for DL-HMTBA.

For comparing the relative activity of methionine sources, Kratzer \& Littell ${ }^{(19)}$ pointed out that the non-linear common plateau asymptotic regression model is not accurate due to the lack of a common plateau. Instead, the authors ${ }^{(19)}$ proposed to use a non-linear separated asymptotic plateau regression. Using published broiler growth data ${ }^{(20)}$ and the non-linear separated asymptotic plateau regression approach, the authors ${ }^{(19)}$ demonstrated that the relative efficiency of methionine sources is dependent on the applied dosage with a better efficiency for DLM at a lower dosage but a better efficiency of HMTBA at higher levels.

However, one other explanation that could contribute to a better performance effect of DL-HMTBA should be linked to the acidifying potential of this molecule. Indeed, both in vitro and in vivo DL-HMTBA shows effective changes in the gut microbiota as in the case of organic acids ${ }^{(21)}$.

It was striking that low-protein DLM-supplemented chickens were consistently characterised by the lowest body weights and the highest proportional heart weights of all 
Table 7. Plasma triiodothyronine $\left(\mathrm{T}_{3}\right)$ and corticosterone levels of 4- and 6-week-old broiler chickens raised on diets with low $(18.3 \%)$ or high $(23.2 \%)$ crude protein (CP) content and supplemented with $0.25 \%$ DL-methionine (DLM) or 0.25\% DL-2-hydroxy-4-methylthiobutanoic acid (DL-HMTBA)

(Mean values with their pooled standard errors, $n$ 15/dietary treatment)

\begin{tabular}{|c|c|c|c|c|c|}
\hline & \multirow[b]{2}{*}{ Met source } & \multicolumn{2}{|c|}{$\mathrm{T}_{3}(\mathrm{ng} / \mathrm{ml})$} & \multicolumn{2}{|c|}{ Corticosterone $(\mathrm{ng} / \mathrm{ml})$} \\
\hline & & Week 4 & Week 6 & Week 4 & Week 6 \\
\hline \multicolumn{6}{|l|}{ Protein level (\%) } \\
\hline \multirow[t]{2}{*}{18.3} & DLM & 1.97 & $0.79^{b}$ & 8.44 & $6 \cdot 49^{a, b}$ \\
\hline & DL-HMTBA & 1.71 & $1.80^{\mathrm{a}}$ & 6.03 & $8 \cdot 10^{\mathrm{a}, \mathrm{b}}$ \\
\hline \multirow[t]{2}{*}{$23 \cdot 2$} & DLM & 1.86 & $1 \cdot 19^{a, b}$ & $6 \cdot 88$ & $8 \cdot 33^{\mathrm{a}}$ \\
\hline & DL-HMTBA & 1.36 & $1 \cdot 16^{a, b}$ & 3.81 & $3.41^{\mathrm{b}}$ \\
\hline Pooled SEM & & 0.06 & 0.11 & 0.62 & 0.67 \\
\hline \multicolumn{6}{|l|}{ Main effects } \\
\hline \multirow[t]{2}{*}{ Protein level } & $18 \cdot 3$ & $1.84^{\mathrm{a}}$ & 1.26 & 7.23 & 7.24 \\
\hline & 23.2 & $1 \cdot 61^{\mathrm{b}}$ & 1.18 & 5.35 & 5.87 \\
\hline \multirow{2}{*}{ Met source } & DLM & $1.91^{\mathrm{a}}$ & 0.99 & $7 \cdot 66^{\mathrm{a}}$ & 7.41 \\
\hline & DL-HMTBA & $1.54^{b}$ & 1.46 & $4.92^{\mathrm{b}}$ & 5.58 \\
\hline \multicolumn{6}{|l|}{ Probabilities } \\
\hline & Protein level & 0.0030 & NS & NS & NS \\
\hline & Met source & 0.0006 & NS & 0.0250 & NS \\
\hline & Interaction & NS & 0.0120 & NS & 0.0130 \\
\hline
\end{tabular}

treatment groups. Based on the proportional growth theory, and given the fact that the heart is an early maturing organ (allometric b-coefficient is approximately 0.85$)^{(22)}$, it is understandable that lighter chickens have a higher proportional heart weight compared with that of heavier animals. However, it cannot be excluded that the supplement source per se could have an effect on heart tissue development.

\section{Redox balance}

The level of TBARS in the plasma is an indicator of (cell membrane) lipid peroxidation (reviewed by Benzie ${ }^{(23)}$ ). The dietary protein content had no significant effect on plasma TBARS levels whereas DL-HMTBA supplementation resulted in a consistently lower lipid peroxidation compared with DLM supplementation within the high protein groups. This favourable effect is not due to an elevation in the total antioxidant capacity of the plasma, as the FRAP capacity of the plasma of DL-HMTBA-supplemented chickens was sometimes lower than that of the DLM-supplemented chickens. Ceruloplasmin is a Cu-containing acute-phase protein with powerful antioxidant capacities (e.g. Park et al. ${ }^{(24)}$ ). It is hence inferred that elevated circulating ceruloplasmin levels will contribute to a better antioxidant defence. However, DL-HMTBAsupplemented chickens were clearly characterised by lower plasma ceruloplasmin levels. Furthermore, uric acid is also recognised as an important antioxidant in mammals ${ }^{(25)}$, and especially in birds ${ }^{(26)}$. Still, in the present study, there is no consistent indication that this antioxidant is involved in the lower lipid peroxidation status of DL-HMTBA-supplemented chickens, as there were no significant differences in circulating uric acid levels between DL-HMTBA- and DLM-supplemented chickens, except at 6 weeks of age, as high-protein DLHMTBA-supplemented chickens were characterised by the highest plasma uric acid levels. Therefore, the non-enzymatic antioxidant capacity of the plasma is not likely to be the predominant causal mechanism for the reduced lipid peroxidation status of the DL-HMTBA-supplemented chickens, except maybe within the high-protein treatment. There is also no direct evidence that the enzymatic antioxidant capacity of the plasma is involved. Based on plasma SOD activity, there were no statistical differences between DL-HMTBAand DLM-supplemented chickens except for a tendency towards higher SOD activity for DL-HMTBA chickens at 6 weeks of age. However, it must be recognised that SOD is only one of the many ROS-scavenging enzymes besides catalase and GSH-recycling enzymes ${ }^{(27)}$.

As there is no clear evidence that the non-enzymatic antioxidant defence mechanisms are responsible for the lower lipid peroxidation status of DL-HMTBA-supplemented chickens, it might be DL-HMTBA itself. Indeed, compared with DLM, DL-HMTBA is more efficiently converted to cysteine as a constituent of taurine and $\mathrm{GSH}^{(14)}$. These sulphur-containing compounds are very powerful antioxidants ${ }^{(7)}$, and a more efficient synthesis and hence higher availability could definitely contribute to an ameliorated ROS-scavenging system. This hypothesis is sustained by the tendency towards higher amounts of total GSH in the liver of 6-week-old low-protein DL-HMTBA-supplemented chickens. It is important to note that this elevation is entirely due to an increase in reduced GSH (representing the major portion of GSH) and not in GSSG. It can be postulated that the favourable redox balance due to DL-HMTBA supplementation to low-protein diets is now mediated by the GSH system and not so much by uric acid as in the case of high-protein diets.

Both Msr-A and -B are responsible for reduction in oxidised methionine residues and therefore are recognised as primary antioxidant defence mechanisms ${ }^{(8)}$. As far as we are aware of, this is the first time that hepatic gene expression of Msr-A has been measured in an avian species. Although we could not observe any treatment differences in Msr-A mRNA levels, this does not mean that the recycling of methionine 
is not an important mechanism in these species. More research is needed to elucidate the contribution of Msr-A in the oxidant defence mechanism of non-mammalian species.

On the other hand, it could also be that DL-HMTBA reduces the production of ROS and hence cellular damage. Indeed, DL-HMTBA-supplemented chickens were also characterised by significantly lower plasma $\mathrm{T}_{3}$ levels, at least at 4 but not at 6 weeks of age. Lower plasma $\mathrm{T}_{3}$ levels are indicative for lower heat production ${ }^{(13)}$, thus less oxygen consumption and consequently reduced formation of free oxygen radicals. Furthermore, DL-HMTBA-supplemented chickens had significantly lower circulating corticosterone levels. As corticosterone is a powerful trigger of oxidative stress as reflected in enhanced lipid peroxidation ${ }^{(11,12)}$, the lower plasma corticosterone levels could also partially explain the reduced TBARS levels in these birds. It remains to be established how DL-HMTBA could directly affect glucocorticoid metabolism.

In conclusion, reducing the dietary protein content of broiler diets resulted in an impaired zootechnical performance compared with that of broilers reared on a higher dietary protein level. However, supplementation of the DLM hydroxy analogue, DL-HMTBA, to the low-protein diet alleviated these negative effects to a certain extent. In addition, the lowprotein DL-HMTBA-supplemented chickens displayed better antioxidant status as reflected in lower lipid peroxidation probably due to their somewhat higher hepatic concentrations of total and reduced GSH levels compared with their DLM counterparts. On the other hand, within the high protein levels, uric acid might be an important antioxidant to explain the lower lipid peroxidation of high-protein DL-HMTBAsupplemented chickens. The present study indicates that there are interactions between dietary protein content and supplementation of DLM (analogues) with respect to broiler performance and redox status, also suggesting a causal link between these traits.

\section{Acknowledgements}

The present study was supported by the Rhodimet Research Grant 2006, which was awarded to the Laboratory for Livestock Physiology, Immunology and Genetics, Katholieke Universiteit Leuven, by Adisseo, France SAS. J. B., E. D., Q. S., P.-A. G. and Y. M. contributed to the conception and design of the study and final interpretation of the data. Q. S., H. W. and N. E. were responsible for the planning of the study and collection of the data. Y. L. was responsible for the molecular analysis of samples. All authors participated in the writing of the final draft of the manuscript and agreed with the final format. The diligent technical assistance of I. Vaesen is gratefully acknowledged. The authors state that there are no conflicts of interest.

\section{References}

1. Sauer N, Emrich K, Piepho H-P, et al. (2008) Meta-analysis of the relative efficiency of methionine-hydroxy-analoguefree-acid compared with DL-methionine in broilers using nonlinear mixed models. Poult Sci 87, 2023-2031.
2. Zhang LB \& Guo YM (2008) Effects of liquid DL-2-hydroxy-4methylthio buanoic acid on growth performance and immune responses in broiler chickens. Poult Sci $\mathbf{8 7}$, $1370-1376$.

3. Garcia Neto M, Pesti GM \& Bakalli RI (2000) Influence of dietary protein level on the broiler chicken's response to methionine and betaine supplements. Poult Sci 79, 1478-1484.

4. Takahashi K, Konashi S \& Akiba Y (1994) The effects of dietary methionine and dispensable amino acid supplementation on abdominal fat deposition in male broilers. Anim Sci Tech 65, 244-250.

5. Vieira SL, Lemme A, Goldenberg DB, et al. (2004) Responses of growing broilers to diets with increased sulfur amino acids to lysine ratios at two dietary protein levels. Poult Sci $\mathbf{8 3}$, $1307-1313$.

6. Fatufe AA \& Rodehutscord M (2005) Growth, body composition, and marginal efficiency of methionine utilization are effected by nonessential amino acid nitrogen supplementation in male broiler chicken. Poult Sci 84, $1584-1592$.

7. Métayer S, Seiliez I, Collin A, et al. (2008) Mechanisms through which sulfur amino acids control protein metabolism and oxidative status. J Nutr Biochem 19, $207-215$

8. Stadtman ER, Van Remmen H, Richardson A, et al. (2005) Methionine oxidation and aging. Biochim Biophys Acta 1703, 135-140.

9. Swennen Q, Decuypere E \& Buyse J (2007) Implications of dietary macronutrients for growth and metabolism of broiler chickens. World's Poult Sci J 63, 541-556.

10. Carsia RV, Weber H \& Lauterio TJ (1988) Protein malnutrition in the domestic fowl induces alterations in adrenocortical function. Endocrinology 122, 673-680.

11. Lin H, Decuypere E \& Buyse J (2004) Oxidative stress induced by corticosterone administration in broiler chickens (Gallus gallus domesticus). 1. Chronic exposure. Comp Biochem Physiol B Biochem Mol Biol 139, 737-744.

12. Lin H, Decuypere E \& Buyse J (2004) Oxidative stress induced by corticosterone administration in broiler chickens (Gallus gallus domesticus). 2. Short-term effect. Comp Biochem Physiol B Biochem Mol Biol 139, 745-751.

13. Buyse J, Decuypere E, Berghman L, et al. (1992) The effect of dietary protein content on episodic growth hormone secretion and on heat production of male broilers. Br Poult Sci 33, 1101-1109.

14. Martin-Venegas R, Geraert P-A \& Ferrer R (2006) Conversion of the methionine hydroxy analogue, DL-2-hydroxy-(4methylbio) butanoic acid, to sulphur-containing amino acids in the chicken small intestine. Poult Sci 85, 1932-1938.

15. Darras VM, Kotanen SP, Geris KL, et al. (1996) Plasma thyroid hormone levels and iodothyronine deiodinase activity following an acute glucocorticoid challenge in embryonic compared with posthatch chickens. Gen Comp Endocrinol 104, 203-212.

16. Sugiyama T, Kawarada Y \& Hirasawa F (2000) Ceruloplasmin. In Experimental Protocols for Reactive Oxygen and Nitrogen Species, pp. 111-113 [N Taniguchi and JMC Gutteridge, editors]. New York: Oxford University Press.

17. De Boever S, Vangestel C, De Backer P, et al. (2008) Identification and validation of housekeeping genes as internal control for gene expression in an intravenous LPS inflammation model in chickens. Vet Immunol Immunopathol 122, 312-317.

18. Vazquez-Anon M, Kratzer D, Gonzalez-Esquerra $\mathrm{R}$, et al. (2006) A multiple regression model approach to contrast 
the performance of 2-hydroxy-4-methylthiobutanoic acid and DL-methionine supplementation tested in broiler experiments and reported in the literature. Poult Sci $\mathbf{8 5}, 693-705$.

19. Kratzer DD \& Littell RC (2006) Appropriate statistical methods to compare dose responses of methionine sources Poult Sci 85, 947-954.

20. Shutte JB \& De Jong J (1996) Biological efficacy of DL-methionine hydroxyl analog free acid compared to DL-methionine hydrox analog free acid in broiler chicks as determined by performance and breast meat yield. Agribiol Res 49, 74-82.

21. Mercier Y, Francesch M, Pérez de Rozas A, et al. (2007) Effects of methionine sources and NSP enzymes on broiler gut microflora. 16th European Symposium of Poultry Nutrition, August 26-30, 2007 Strasbourg, France, pp. 577-580.

22. Govaerts T, Room G, Buyse J, et al. (2000) Early and temporary quantitative food restriction of broiler chickens. 2 . Effects on allometric growth and growth hormone secretion. Br Poult Sci 41, 355-362.

23. Benzie IFF (1996) Lipid peroxidation: a review of causes, consequences, measurement and dietary influences. Int $J$ Food Sci Nutr 47, 233-261.

24. Park YS, Suzuki K, Taniguchi N, et al. (1999) Gluthatione peroxidase-like activity of caeruloplasmin as an important lung antioxidant. FEBS Lett 458, 133-136.

25. Hellstein Y, Tullson PC, Richter EA, et al. (1997) Oxidation of urate in human skeletal muscle during exercise. Free Radic Biol Med 22, 169-174.

26. Simoyi MF, Dyke KV \& Klandorf H (2002) Manipulation of plasma uric acid in broiler chicks and its effect of leucocyte oxidative injury. Am J Physiol 282, R791-R796.

27. Bottje WG \& Wideman RF Jr (1995) Potential role of free radicals in the pathogenesis of pulmonary hypertension syndrome. Poult Avian Biol Rev 6, 211-231. 